\title{
Influence of chitosan addition on the mechanical and antibacterial properties of carrot cellulose nanofibre film
}

\author{
Monika Szymańska-Chargot $(\mathbb{D} \cdot$ Monika Chylińska • Giorgia Pertile • \\ Piotr M. Pieczywek · Krystian J. Cieślak • Artur Zdunek - Magdalena Frąc
}

Received: 15 May 2019/Accepted: 12 September 2019/Published online: 18 September 2019

(C) The Author(s) 2019

\begin{abstract}
Films of carrot cellulose nanofibrils (CCNFs) with the addition of low-viscosity chitosan (CHIT) were prepared by the vacuum filtration. The chitosan content in the films varied from 9 to $33 \%$ (dry wt. basis). The surface morphology of the films was investigated by scanning electron microscopy, and it was found that chitosan was dispersed in the CCNF matrix. The interaction between CCNFs and CHIT was evaluated in terms of Fourier transform infrared spectroscopy (FTIR). The obtained results suggested physical interactions rather than hydrogen bonding between CCNFs and CHIT. This finding also supports the results of the water wettability experiment. The addition of chitosan to the nanocellulose matrix causes an increase in the water contact angle, i.e., the surface of the composites becomes more hydrophobic. This increase is probably connected to an interaction
\end{abstract}

Electronic supplementary material The online version of this article (https://doi.org/10.1007/s10570-019-02755-9) contains supplementary material, which is available to authorized users.

M. Szymańska-Chargot $(\bowtie) \cdot$ M. Chylińska ·

G. Pertile · P. M. Pieczywek · A. Zdunek · M. Frąc

Institute of Agrophysics, Polish Academy of Sciences,

Doswiadczalna 4, 20-290 Lublin 27, Poland

e-mail: m.szymanska@ipan.lublin.pl

\section{K. J. Cieślak}

Faculty of Environmental Engineering, Institute of Renewable Energy Engineering, Lublin University of

Technology, Nadbystrzycka 40B, Lublin, Poland between nanocellulose and chitosan forming a denser structure. Analyses of thermal properties showed that the composites are stable under high temperature, and the degradation occurred above $300{ }^{\circ} \mathrm{C}$. It was found that the addition of CHIT to CCNF matrices caused a decrease in the Young's modulus - the higher that the concentration of chitosan in the composite was, the lower the Young's modulus (decreased from $14.71 \mathrm{GPa}$ for CCNFs to $8.76 \mathrm{GPa}$ for $\mathrm{CCNF} /$ CHIT_5). Additionally, the tensile strength of composites, i.e., the maximum force that causes a fracture decreased after the addition of chitosan (decreased from $145.83 \mathrm{MPa}$ for CCNFs to $129.43 \mathrm{MPa}$ for CCNF/CHIT_5). The results indicated the highest inhibitory effect of the investigated composites against E. coli and S. epidermidis. Whereas M. luteus was inhibited only by the higher concentration of chitosan in the tested composites, inhibition was not found against $C$. krissii and all tested filamentous fungi. 


\section{Graphic abstract}
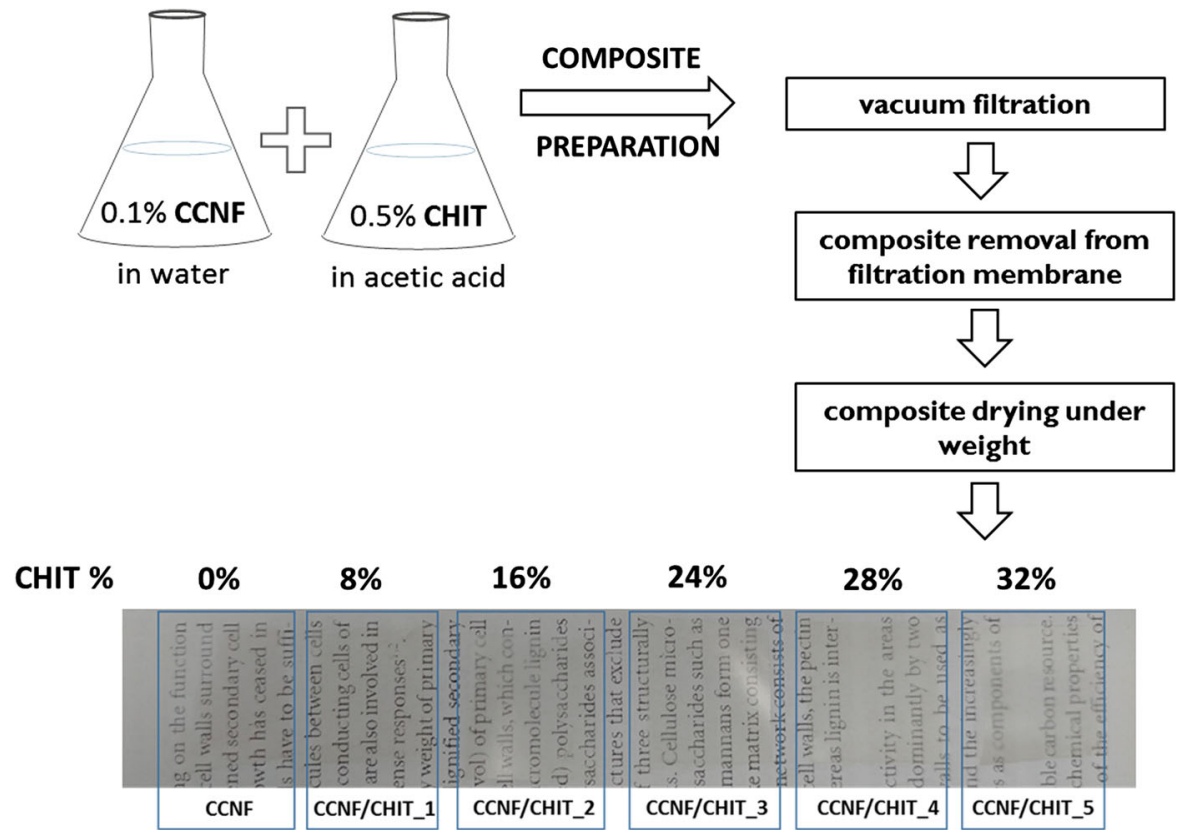

\section{$\Rightarrow$ Thermal properties (DSC) $\Rightarrow$ Antibacterial properties}

\section{$\Rightarrow$ Structure (FT-IR) $\quad \Rightarrow$ Mechanical properties}

Keywords Nanocellulose $\cdot$ Chitosan $\cdot$ Antibacterial properties $\cdot$ Mechanical properties $\cdot$ Thermal properties

\section{Introduction}

Cellulose and chitin are two of the most abundant polymers on Earth. Both are biodegradable and biocompatible and are derived from natural renewable sources (Chen 2017; Dufresne 2012). Chitin, a linear polysaccharide of $\beta-1,4-N$-acetylglucosamine, is a component of the fungal cell wall and exoskeletons of insects or crustaceans (Chen 2017). Due to its high crystallinity and strong intermolecular bonds, chitin has low reactivity and is water insoluble. Therefore, to overcome that limitation, after deacetylation, chitosan is obtained. Chitosan is easily dissolved at low $\mathrm{pH}$, and its composites with a wide range of polymers were analysed (Deng et al. 2017). Subsequently, due to their biocompatibility, biodegradability, antibacterial activity, and non-toxicity, their applications were studied in agriculture as a fertilizer, in the food industry as a stabilizer and thickener and in the field of biomedical engineering (de Alvarenga 2011; de Mesquita et al. 2010). The main disadvantage of chitosan composites is their poor mechanical properties (Abdul Khalil et al. 2016). On the other hand, positively charged chitosan is a natural antimicrobial agent against an extensive variety of microorganisms having potential as an antimicrobial agent to improve food safety and quality (Kim et al. 2011). Chitosan's antimicrobial activity is influenced by the type of chitosan, its degree of deacetylation, its degree of polymerization, the tested host, the age of the microbial cell, the source of the medium, the chemical composition of the medium, water activity inside the medium, its concentration and pH of the medium (Dutta et al. 2009; Kong et al. 2010).

Cellulose is a linear polymer of $\beta-1,4$-glucose organized into fibrils, the main constituent of the plant cell wall (Szymańska-Chargot et al. 2011). Cellulose individual fibrils are well known for their unique properties such high mechanical strength, making them comparable to such materials as the Kevlar fibre 
or steel wire (Brinchia et al. 2013; Moon et al. 2011; Wang et al. 2015). Moreover, there is growing research interest in the use of cellulose nanomaterials such as nanofibrillated cellulose and cellulose nanocrystals as eco-friendly fillers and reinforcement for existing composites (Moon et al. 2011; Ämmälä et al. 2013). So far nanocellulose is produced from both forest and agriculture resources (Alemdar and Sain 2008; Chen et al. 2011; Liu et al. 2012, Rambabu et al. 2016; Rahmi and Julinawati 2017). Recently, the possibility of the use of fruit and vegetable pomaces as the source of cellulose was presented (SzymańskaChargot et al. 2017). The cellulose was extracted under mild acid-alkali conditions, and cellulose nanofibres (CNF) were produced by high-intensity ultrasound (Szymańska-Chargot et al. 2017, 2018, 2019). CNF or, in general, nanocellulose films are often called nanopapers due to the analogous production methods with cellulosic-based paper (Klemm et al. 2011; Stark 2016). The CNF films themselves are highly flexible due to the entangled network of ultrathin nanofibrils (Wang et al. 2015). Moreover, CNF-only films have good optical transparency, and the addition of CNF to polymers does not affect the transparency (Abdul Khalil et al. 2016). Once the film is produced, the CNFs cannot be easily spread again in water, although, generally, nanocellulose films are not fully water-resistant. The main advantage of CNF films are small pores as a results of both hydrogen bonding between nanofibrils and high crystallinity, giving materials with good barrier properties useful for packaging materials (Klemm et al. 2011; Stark 2016). However, the material source, morphology, and chemical composition affect the barrier properties of CNF films (Lavoine et al. 2012). Cellulose nanostructures are usually used as a reinforcing agent for several polymers, but they can also be used as matrices for various materials, including films for food-packaging materials (Azeredoa et al. 2017). Rahmi and Julinawati (2017) showed that hydrogen bonds forming between cellulose and chitosan helps to obtain good adhesion between both materials even without using surface modificators or coupling agents (Rahmi and Julinawati 2017). Most of the publication reporting results of chitosan reinforced by cellulose focused on commercial cellulose which unfortunately results in high-cost material (AboElseoud et al. 2018; Hassan et al. 2011, 2016; Trana et al. 2013).
Thus, although the properties and applications of chitosan-cellulose blends and nanocellulose-reinforced chitosan biocomposites have been reported, there is still a lack of publication reporting of the use of cellulose isolated from fruit or vegetable pomace as the composite with chitosan (Abdul Khalil et al. 2016). Previously, it has been shown that films obtained from nanocellulose originating from apple and carrot pomaces present very good mechanical properties (Szymańska-Chargot et al. 2019). The utilization of cellulose isolated from pomaces may result in a lowcost material. Here, cellulose was isolated from carrot pomace, and nanocellulose was obtained by the highintensity ultrasonication method (HIUS) (SzymańskaChargot et al. 2017). Chitosan, although it has interesting antimicrobial properties, is known to have poor mechanical properties. Thus, reinforcing agents are still being searched (Elsabee and Abdou 2013). Nanocellulose, with its biocompatibility, biodegradability and good optical transparency, is the potential reinforcing agent for chitosan films (Fernandes et al. 2010). Thus far, nanocellulose, both in the form of nanofibres or nanowhiskers, was used as the reinforcement of chitosan films (Fernandes et al. 2010; Falamarzpour et al. 2017; Dehnad et al. 2014) and most of the studies are focused on the characterization of their mechanical and antibacterial properties ( $\mathrm{Li}$ et al. 2009; Rahmi and Julinawati 2017; Niu et al. 2018; Toivonen et al. 2015; Wu et al. 2014). Moreover, the addition of nanocellulose could increase the flexibility and thermal stability of chitosan-nanocellulose films compared with native chitosan films (Fernandes et al. 2010). This characteristic of chitosan-CNF films makes them useful for various applications such as electronic devices, medical and antibacterial packaging. However, reports are lacking concerning nanocellulose as the matrix and chitosan as an antimicrobial agent (Hänninen et al. 2018). Here, for the first time, composites based on nanocellulose prepared from carrot cellulose with different concentrations of chitosan from shrimp shells were prepared. The nanocellulose was the base matrix for which chitosan played only a role as an antimicrobial agent. 


\section{Materials and methods}

\section{Materials}

Never-dried cellulose was isolated from carrot pomace prepared in a de-pulping machine with a double-screw shredder (Twin Gear Juice Extractor; Green Star Elite GSE-5000, Anaheim, CA, USA) as described by the method of Szymańska-Chargot et al. (2017). Carrot was purchased in a local grocery shop. Low-viscosity chitosan from shrimp shells was purchased from Sigma Aldrich Poland.

Preparation of nanofibrillated cellulose from carrot pomace

The carrot cellulose nanofibrils (CCNFs) were prepared using high-intensity ultrasonication (Szymańska-Chargot et al. 2019). The ultrasonic homogenizer Vibra Cell VCX 130 (SONICS \& MATERIALS Inc.) with a net power output equal to 130 watts and a frequency of $20 \mathrm{kHz}$ was used. The processor was equipped with a 6-mm-diameter probe with a maximum oscillation amplitude equal to $114 \mu \mathrm{m}$. The sonication system contained a temperature probe, and, to avoid heating of the sample, an ice-bath was used. The operating amplitude of the ultrasonic homogenizer was maintained to $90 \%$ of the nominal amplitude.

Each time, $250 \mathrm{~g}$ of the $0.2 \%$ water suspension of carrot cellulose was prepared. First, the Ultra-Turrax (T10 basic ULTRA TURRAX, IKA) was used for $10 \mathrm{~min}$ to initial disperse the obtained suspensions. Next, the dispersed samples were introduced to ultrasound treatment for $30 \mathrm{~min}$. Thereafter, the sample was divided into two portions and diluted to obtain the $0.1 \mathrm{wt} \%$ dispersion. Each portion was introduced to ultrasound treatment for $30 \mathrm{~min}$. Thereafter, the $0.1 \mathrm{wt} \%$ CCNFs dispersion was obtained. The procedure of CCNFs preparation together with the photo of CCNFs dispersion and AFM height image of the CCNFs is presented in Supplementary Material Fig. 1.

\section{Composite preparation}

To obtain the nanocellulose-chitosan composites, a $0.5 \%$ solution of chitosan (CHIT) in $2 \%$ acetic acid was prepared. Subsequently, the following weight proportions of solutions were prepared $(\mathrm{CCNF} /$ CHIT): 10:1 (CCNF/CHIT_1), 10:2 (CCNF/CHIT_2), 10:3 (CCNF/CHIT_3), 10:4 (CCNF/CHIT_4) and 10:5 (CCNF/CHIT_5) to obtain a chitosan concentration from 9 to $33 \%$ of the resulting composite dry weight. The accurate weight proportions of CHIT and CCNFs in each composite are presented in Table 1. Next, $250 \mathrm{~g}$ of the obtained mixtures and the pure CCNFs dispersion ( $0.1 \%$ in water) were filtered under vacuum (0.6 bar) in the system comprising the vacuum pump Basic 36 (AgaLabor, Poland) and filtration set (1000-mL flask, funnel and clamp; Chempur, Poland). In every case, a $0.65-\mu \mathrm{m}$-porediameter PDF membrane filter (EMD Millipore ${ }^{\mathrm{TM}}$ Durapore $^{\mathrm{TM}} ; \varphi=90 \mathrm{~mm}$ ) was used. The filtration process lasted approximately $20 \mathrm{~h}$ and was followed by water rinsing to neutral $\mathrm{pH}$ to remove acetic acid from composites. Thereafter, each composite was dried under a load of $7 \mathrm{~kg}$ for $72 \mathrm{~h}$. The final weight of each composite was $0.25 \mathrm{~g}$.

\section{FTIR}

FTIR spectra were collected in the Nicolet 6700 Fourier Transform infrared spectrometer (FTIR, ThermoScientific, Waltham, MA, USA). The Smart iTR ATR sampling accessory was used. The composite films were directly placed on the ATR crystal and measured over the range of $4000-650 \mathrm{~cm}^{-1}$. For each material, 3 samples under the same conditions were examined. For each sample, 200 scans were averaged with a spectral resolution of $4 \mathrm{~cm}^{-1}$. Next, for a given material, the final average spectrum was calculated. These spectra were normalized to 1.0 at $1017 \mathrm{~cm}^{-1}$ (COH stretching vibration). The differential spectra between the CCNF/CHIT spectra and CCNFs spectrum were calculated to determine and highlight differences between composites. All the spectra manipulation was carried out using The Origin Pro 8.5 (OriginLab Corporation, USA).

\section{Differential scanning calorimetry (DSC)}

DSC analysis was performed using a TA Instruments DSC 250 system (Waters, DE, USA) and 5-10 mg of the film's samples sealed in aluminium pans and a 20-400 ${ }^{\circ} \mathrm{C}$ heating increase under a nitrogen flux of $50 \mathrm{~mL} \mathrm{~min}{ }^{-1}$; the heating rate was $10^{\circ} \mathrm{C} \mathrm{min}{ }^{-1}$. Analyses were preceded by scans in the heat-cool- 
Table 1 Weight proportions of CHIT and CCNFs in each composite

\begin{tabular}{llll}
\hline COMPOSITE & CHIT $(\mathrm{g})$ & CCNFs $(\mathrm{g})$ & Content of CHIT in composite (\%) \\
\hline CCNFs & 0.000 & 0.250 & 0.0 \\
CCNF/CHIT_1 & 0.023 & 0.227 & 9.1 \\
CCNF/CHIT_2 & 0.042 & 0.208 & 16.7 \\
CCNF/CHIT_3 & 0.058 & 0.192 & 23.1 \\
CCNF/CHIT_4 & 0.071 & 0.179 & 28.6 \\
CCNF/CHIT_5 & 0.083 & 0.167 & 33.3 \\
\hline
\end{tabular}

heat mode with a maximum temperature at $200{ }^{\circ} \mathrm{C}$ to eliminate water from the sample but not cause its decomposition. The data were analysed using Trios v.4.2.1 (TA Instruments, Waters, DE, USA) software. Enthalpy was determined as the area under the peak of the curve in the range $240-380{ }^{\circ} \mathrm{C}$.

Mechanical properties of composites

All the composites under the study were tested for tensile strength, Young's modulus and elongation at break. Samples of composites were prepared as rectangular strips, with a length of $40 \mathrm{~mm}$ and a width of $\sim 2 \mathrm{~mm}$. Precise measurements of the sample width were carried out using the Olympus SZX16 (Olympus Corporation, Japan) microscope with an SDF PLAPO 0.5 XPF lens, equipped with a DFK 51BU02.H digital camera (The Imaging Source Europe $\mathrm{GmbH}$, Bremen, Germany). Image resolution was equal to $8.26 \mu \mathrm{m}$ per pixel. The width of each sample was calculated as the mean value from three measurements. The thickness of each sample was measured using a digital micrometre BAKER IP54 (Baker Gauges India Private Limited, India) with a measurement accuracy equal to $0.001 \mathrm{~mm}$. The composite film strips were subjected to uniaxial tensile testing using a miniature tensile stage (Deben Microtest, Suffolk, UK). The initial gap between grips was equal to $10 \mathrm{~mm}$. Mechanical experiments were carried out up to sample rupture with a deformation speed of $0.2 \mathrm{~mm} \mathrm{~min}^{-1}$. Tensile force and elongation of the sample were recorded and converted into stress and strain, respectively. Stress was determined as the ratio of the tensile force to the sample cross-sectional area. The strain was defined as the ratio of the sample elongation to its initial length. The Young's modulus was determined as the slope of the longest linear part of the stress-strain curve. Mechanical tests were repeated ten times for each composite film.

\section{Wettability}

The contact angles of 5- $\mu \mathrm{L}$ water droplets on the film's surface attached to microscopic slides by double-sided tape (T: $23.5 \pm 0.1{ }^{\circ} \mathrm{C}$; RH: $31.6 \%$ ) were measured. The Rame Hart 200Std goniometer was used. Measurements were performed for 10 min with 1-s time intervals. A longer measurement did not give any reliable results, and it was decided to be completed after $10 \mathrm{~min}$. Each point on the plot corresponds to the mean value of the left and right contact angle at a given point in time. Additionally, photos of droplets were taken immediately after droplet setting, after 10 and after 20 min (Supplementary Material Fig. 2).

\section{Scanning electron microscopy (SEM)}

The morphology of the CCNFs and CCNF/CHIT composite surface was examined by scanning electron microscopy (SEM; Hitachi SU3500) at $1.5 \mathrm{kV}$ under high-vacuum conditions. The small cut of each sample was applied on the aluminium stage covered by carbon tape. Next, the samples were coated with an ultrathin gold layer $(\mathrm{Au})$ using an ion-sputtering machine (Cressington Sputter Coater 108 Auto).

Composite antibacterial properties

\section{Microorganism pre-culturing}

To analyse the inhibition of CCNFs and CHIT composites, four bacterial species (Staphylococcus epidermidis, Escherichia coli, Bacillus cereus, and Micrococcus luteus), one yeast species (Candida krissii), and four filamentous fungal species (Botrytis cinerea, Neosartorya fischeri, Petriella setifera and Fusarium oxysporum) were selected from the microorganism collection of the Laboratory of Molecular and Environmental Microbiology, Institute of 
Agrophysics, Polish Academy of Sciences (Lublin, Poland). The information about the microorganisms and conditions of pre-culturing were described in Supplementary Material SM Table 1. Micrococcus luteus, yeast and all filamentous fungi were incubated at $28{ }^{\circ} \mathrm{C}$, whereas the remaining bacterial strains were incubated at $37{ }^{\circ} \mathrm{C}$. All microorganisms were shaken at $180 \mathrm{rpm}$ for $24 \mathrm{~h}$ and $72 \mathrm{~h}$ for bacteria and fungi, respectively.

\section{Antimicrobial assay through the inhibition zone test}

The antimicrobial properties were determined by measurements of the inhibition zone of bacteria and fungi using the modified Kirby-Bauer Disk diffusion susceptibility test protocol (Hudzicki 2009). For each medium (Supplementary materials Table 1), the $\mathrm{pH}$ was corrected to 6 using $\mathrm{HCl}$. The Petri dishes were divided into four equal parts. Each quarter was tested with CCNFs and CCNF/CHIT_1-5 films. Each composite cut with a diameter of $5 \mathrm{~mm}$ was prepared using a paper puncher. To confirm the antimicrobial activity of chitosan, the spreading on each medium of $300 \mu \mathrm{L}$ $0.25 \%(\mathrm{w} / \mathrm{v})$ of chitosan dissolved in broth medium or adding $0.25 \%(\mathrm{w} / \mathrm{v})$ of chitosan to the medium before sterilization was used. Each treatment was prepared in three replicates. Each Petri dish was inoculated with $300 \mu \mathrm{L}$ and $500 \mu \mathrm{L}$ of the bacterial and fungal cell suspensions, respectively. The initial number of bacteria and fungi was approximately of $10^{7}-10^{8}$ CFU mL ${ }^{-1}$, with an OD of 1 at $610 \mathrm{~nm}$ and $750 \mathrm{~nm}$ for bacteria and fungi, respectively. The OD was measured using the Infinite ${ }^{\circledR}$ M200PRO spectrophotometer (Tecan, Switzerland). Inoculated Petri dishes were incubated at $28{ }^{\circ} \mathrm{C}$ for Micrococcus luteus, yeast and all filamentous fungi and $37^{\circ} \mathrm{C}$ for other bacteria. The diameter of the inhibition zone (in $\mathrm{mm}$ ) was measured every $24 \mathrm{~h}$ for 4 days. For the fungi, during the experiment, the inhibition of chitosan applied alone was not observed; therefore, a higher concentration of chitosan at $1 \%(\mathrm{w} / \mathrm{v})$ was tested. The procedure was the same as described above, but measurements of the inhibition zone were performed every $24 \mathrm{~h}$ for 7 days.

\section{Inhibition test using the optical density (OD)}

The inhibitory effects of different concentrations of CCNFs and chitosan on microorganism growth were estimated by the optical density (OD) measurements of each culture. For antimicrobial analysis, aliquots of $200 \mu \mathrm{L}$ of each bacterial or fungal suspension (at 1 OD) were added to each well together with the film comprising different concentrations of CCNFs and CHIT or CHIT alone. Furthermore, to avoid overestimation of the $\mathrm{OD}$, during plate reading, each composite (CCNF, CCNF/CHIT) and chitosan alone were loaded into separate wells without the inoculum as a negative control. The results were standardized by subtracting the OD of the composite (CCNF/CHIT) or chitosan alone from the OD of the inoculum with the different composites. The study was performed in three replicates. The optical density was read at $610 \mathrm{~nm}$ and $750 \mathrm{~nm}$ for bacteria and fungi, respectively, using the Infinite ${ }^{\circledR}$ M200PRO spectrophotometer (Tecan, Switzerland) every $24 \mathrm{~h}$ for 7 days.

Statistical analysis

To illustrate the significant differences, one- and twoway ANOVA were performed between the optical density and microorganisms, time and treatment. Subsequently, significant differences were calculated by post hoc analysis using Tukey's test. Moreover, Pearson's correlation between the concentration of chitosan, nanofibrils, and water availability of each film was assessed. All the statistical analyses were performed using STATISTICA 13.1 software (StatSoft, Inc., USA).

\section{Results}

Structure and morphology of composites

The CCNFs used in this study was produced in the form of nanofibrils. Previously, it was shown that the average diameter of CCNFs was $3.31 \mathrm{~nm}$, and the length did exceed $1 \mu \mathrm{m}$. The crystallinity degree, as evaluated by XRD, was $80 \%$ (Szymańska-Chargot et al. 2019).

The transparency of the CCNF/CHIT films is illustrated in Fig. 1a. Only in the case of the films with the highest percentage of chitosan content (CCNF/CHIT_5) did visible blurring of the image occur. The morphology of the composite was evaluated by SEM (Fig. 1b). The CCNF film SEM micrograph shows very uniform and homogeneous material. 
Fig. 1 Photograph of $\mathrm{CCNF} / \mathrm{CHIT}$ cut strip films indicating optical transparency (a). SEM images of $\mathrm{CCNF} / \mathrm{CHIT}$ composites (b)

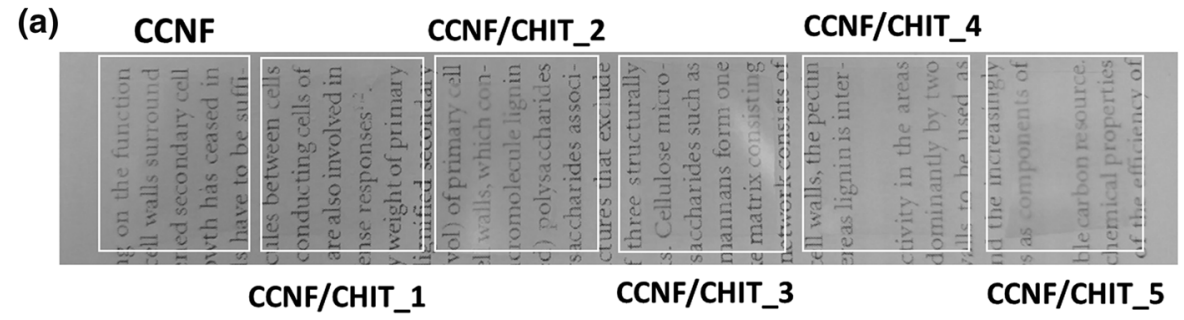

(b)

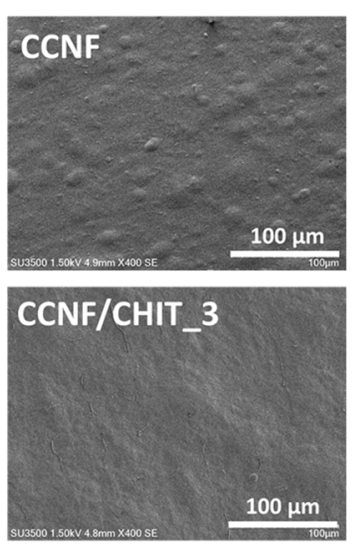

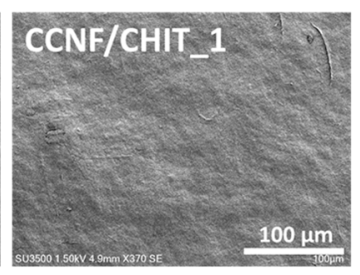
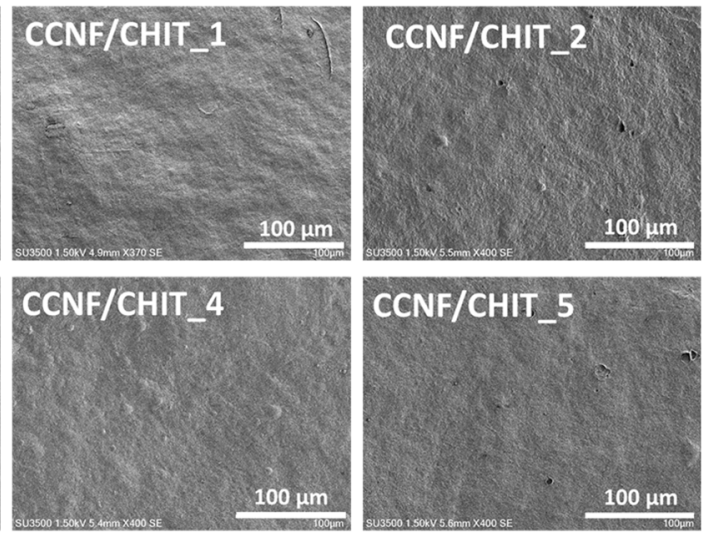

In the case of the addition of chitosan, the random orientation and good dispersion of chitosan in CCNF matrices are visible. These results indicate the good compatibility between both polymers, resulting in homogeneous material.

FTIR analysis was used to characterize the effect of chitosan addition on CCNF films. The shifts of FTIR bands are usually related to interactions between molecules. The spectra of pure CCNF and chitosan were used as reference one. The spectra of each variation of $\mathrm{CCNF} / \mathrm{CHIT}$ blend and CCNFs were shown to highlight differences among the sample structures (Fig. 2). The CCNFs spectra contain typical bands for cellulose. The region between 3600 and $3200 \mathrm{~cm}^{-1}$ is assigned to $\mathrm{O}-\mathrm{H}$ stretching vibrations with the maximum at $3343 \mathrm{~cm}^{-1}$ related to $\mathrm{O}-\mathrm{H}$ vibration due to hydrogen intramolecular bonding (Fig. 2a) (Szymańska-Chargot et al. 2019). The region $3000-2800 \mathrm{~cm}^{-1}$ is related to $\mathrm{C}-\mathrm{H}$ and $\mathrm{C}-\mathrm{H}_{2}$ stretching vibrations. The region $1500-1250 \mathrm{~cm}^{-1}$ is related to $\mathrm{CH}$ deformation and $\mathrm{OH}$ out-of-plane bending vibrations ( $\mathrm{Li}$ and Renneckar 2011; Zhbankov et al. 2002). The region between 1180 and $800 \mathrm{~cm}^{-1}$ has the same pattern for the CCNFs and CHIT samples due to their similar structure. This region is also sensitive for the $\mathrm{C}-\mathrm{O}$ and $\mathrm{C}-\mathrm{C}$ stretching vibrations (Szymańska-
Chargot and Zdunek 2013; Dimzon and Knepper 2015; de Souza Costa Júnior et al. 2009; Krishna Rao et al. 2006). The main characteristic feature is the presence of bands at $1610 \mathrm{~cm}^{-1}$ (overlapped by water band at $1640 \mathrm{~cm}^{-1}$ ) related to carbonyl group stretching, while bands at $1740 \mathrm{~cm}^{-1}$ are related to ester stretching. This results in cellulose oxidation as an outcome bleaching process with sodium hypochlorite (Li and Renneckar 2011; Szymańska-Chargot et al. 2019).

In the chitosan films spectrum (Fig. 2a) region between 3500 and $3250 \mathrm{~cm}^{-1}$ can be assigned to the stretching vibrations of intra- and intermolecular $\mathrm{O}-\mathrm{H}$ and $-\mathrm{CH}_{2} \mathrm{OH}$, but also stretching vibrations of $-\mathrm{NH}_{2}$ $\left(3358 \mathrm{~cm}^{-1}\right)$ and $-\mathrm{NH}$ secondary amide vibrations $\left(3288 \mathrm{~cm}^{-1}\right)$ can be visible. Additionally, region $2960-2870 \mathrm{~cm}^{-1}$ is typical for symmetric and asymmetric vibrations of $\mathrm{C}-\mathrm{H}$ (Khan et al. 2012). The peaks at $1647 \mathrm{~cm}^{-1}, 1583 \mathrm{~cm}^{-1}$ and $1557 \mathrm{~cm}^{-1}$ are due to the amide I, primary amino group and amide II, respectively (Li et al. 2011; Khan et al. 2012).

In the case of CCNF/CHIT composites, some differences can be observed on the FTIR spectra (Fig. 2b). The main differences are related to the presence of bands originated from chitosan amine and amide group vibration: 1647, 1583 and $1557 \mathrm{~cm}^{-1}$. 
Fig. 2 a FTIR spectra of chitosan (CHIT) and CCNFs pure samples. b Spectra of CCNF/CHIT_1-5 composites. Only the most important wavenumbers are listed in the figure

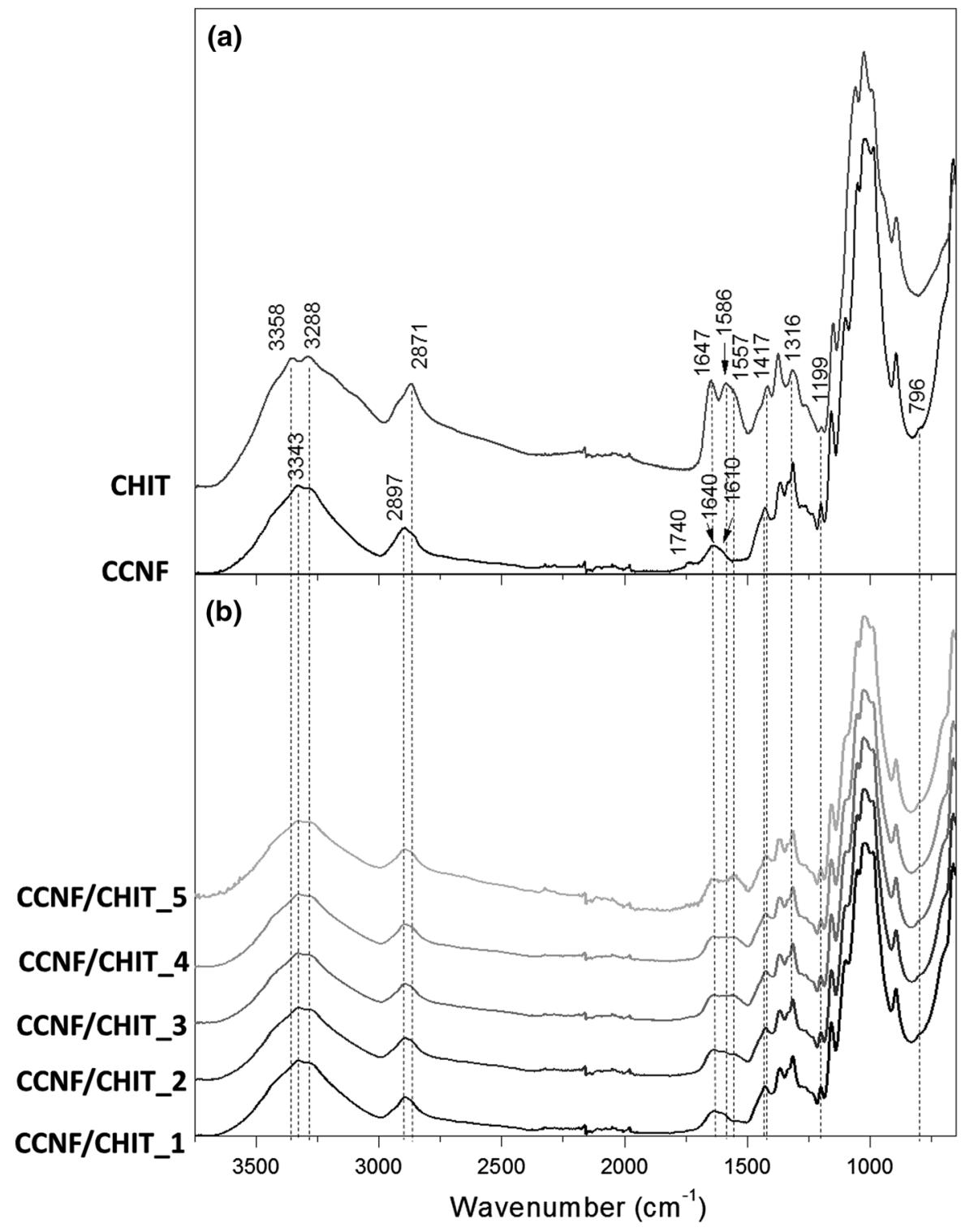

For pure chitosan, the intensity of 1647 and $1586 \mathrm{~cm}^{-1}$ bands were the same. In the case of $\mathrm{CCNF} / \mathrm{CHIT}$, the primary amine band at $1583 \mathrm{~cm}^{-1}$ diminished, and the band related to amide II increased. On the other hand, CCNF/CHIT_5 had higher intensity for the $1583 \mathrm{~cm}^{-1}$ than that for the $1647 \mathrm{~cm}^{-1}$ band. Moreover, in the case of CCNF/CHIT composites, the band connected to the carboxyl ester disappeared. Previously, the band at $3343 \mathrm{~cm}^{-1}$ was related to the hydrogen bonding between nanocellulose and chitosan (Khan et al. 2010). However, here, it is less likely because this band is also present in the pure
CCNFs spectrum (reflecting the intramolecular hydrogen bonds in cellulose; please see ref.: SzymańskaChargot et al. 2019), and, besides that, no visible shift of this band occurred.

\section{Thermal properties}

The differential scanning calorimetry was used to evaluate the thermal properties of the composites. The DSC curves and detailed data are presented in Supplementary Materials Fig. 3 and Table 2. The striking feature is the lack of glass transition for either 
Table 2 Data from DSC curves corresponding to the material decomposition process

\begin{tabular}{lll}
\hline Sample & \multicolumn{2}{l}{ Decomposition } \\
\cline { 2 - 3 } & $\mathrm{T}_{\max }\left({ }^{\circ} \mathrm{C}\right)$ & Enthalpy $(\mathrm{J} / \mathrm{g})$ \\
\hline CCNFs & 349.3 & 91.9 \\
CHIT & 311.0 & 328.2 \\
CCNF/CHIT_1 & 319.9 & 175.4 \\
CCNF/CHIT_2 & 316.9 & 205.9 \\
CCNF/CHIT_3 & 317.5 & 210.2 \\
CCNF/CHIT_4 & 315.7 & 238.6 \\
CCNF/CHIT_5 & 319.0 & 230.6 \\
\hline
\end{tabular}

CCNF/CHIT composites or pure CCNFs and CHIT. Only the thermal decomposition occurred (Supplementary Materials Fig. 3). In the DSC curves, the biopolymer film decomposition is reflected by exothermal peaks with a maximum above $300{ }^{\circ} \mathrm{C}$ and was $349.3{ }^{\circ} \mathrm{C}$ for $\mathrm{CCNF}$ film and $311.0^{\circ} \mathrm{C}$ for CHIT (Nada and Hassan 2000). However, chitosan addition introduced a significant impact on the thermal properties of composites CCNF/CHIT_1-5, and the decomposition temperature ranged from 315.7 to $319.9{ }^{\circ} \mathrm{C}$. A similar situation was observed in the case of the enthalpy calculated for the decomposition process. The addition of chitosan caused an increase in the enthalpy value. For CCNFs, the enthalpy value was equal to $91.9 \mathrm{~J} / \mathrm{g}$; for CHIT, it was $328.2 \mathrm{~J} / \mathrm{g}$; for the mixtures CCNF/CHIT_1-5, this value ranged from 175.4 to $238.6 \mathrm{~J} / \mathrm{g}$ and increased with the increasing amount of chitosan.

\section{Wettability}

Figure 3 shows air-water contact angles changes in time. Noticeable differences between the contact angles values were observed already after droplet setting. This value was equal to $64^{\circ}$ for CCNFs, approximately $91^{\circ}-93^{\circ}$ for CCNF/CHIT_1-4 composites, and $98^{\circ}$ for the CCNF/CHIT_5 sample. A higher chitosan content and lower wettability of materials was observed. During the measurements, the drops slowly spilled over the materials. After $10 \mathrm{~min}$ of contact, the angles decreased as follows: to $43^{\circ}$ for CCNF, to $63^{\circ}$ for CCNF/CHIT_ $1-3$, to $66^{\circ}$ for CCNF/CHIT_4 and to $73^{\circ}$ for CCNF/CHIT_5. An explanation is that the increasing interaction of CHIT with CCNFs leads to less available hydroxyl groups, resulting in a lower hydrophilic nature of CCNF films (Deng et al. 2017). Photographs of water droplets on composites taken at 10 and $20 \mathrm{~min}$ are presented as Supplementary material Fig. 2.

\section{Mechanical properties}

Figure 4 presents typical tensile stress-strain curves of $\mathrm{CCNF} / \mathrm{CHIT}$ composites with varying CHIT concentrations. The influence of chitosan addition on the mechanical properties of nanocellulose film is presented in Table 3. Young's modulus is a measure of the stiffness of a solid material in the linear elasticity model. Young's modulus describes the elastic properties of material the higher is the value, the less deformation is created in the material under tensile or compressive stress. The highest Young's modulus was obtained for the pure CCNF film, and it was $14.71 \pm 1.27 \mathrm{GPa}$ (Table 2). The addition of chitosan caused the decrease in the Young's modulus of composite films from $10.82 \mathrm{GPa}$ for CCNF/CHIT_1 to $8.76 \mathrm{GPa}$ for CCNF/CHIT_5, indicating that chitosan acts similar to plasticizer and enhances the ductility of nanocellulose itself. Other parameters are the yield strength and strain. The yield strength is highest for the CCNF sample with a value of $92.90 \pm 15.05 \mathrm{MPa}$, whereas for the composites, the yield strain was the lowest in this case. The addition of chitosan caused a decrease in the yield strength with a simultaneous increase in the yield strain (Table 2). The pure nanocellulose sample has a longer elastic region than that of the composites of CCNFs and CHIT. The highest tensile strength was obtained for the CCNFs sample, and the addition of chitosan caused a decrease in the tensile strength; however, this change was not large. The maximum strain compared with the CCNFs sample (report maximum value for CCNF/CHIT_4) was increased for composites after the addition of chitosan and reached a value above $2 \%$.

Antimicrobial assay through the inhibition zone test

The inhibition zone analysis showed that the highest inhibition was observed for Escherichia coli and Staphylococcus epidermidis (Table 4). For these two bacteria, an inhibition effect in all tested films in the 
Fig. 3 Change in time of the contact angle

Fig. 4 Typical tensile strain curves of the test films
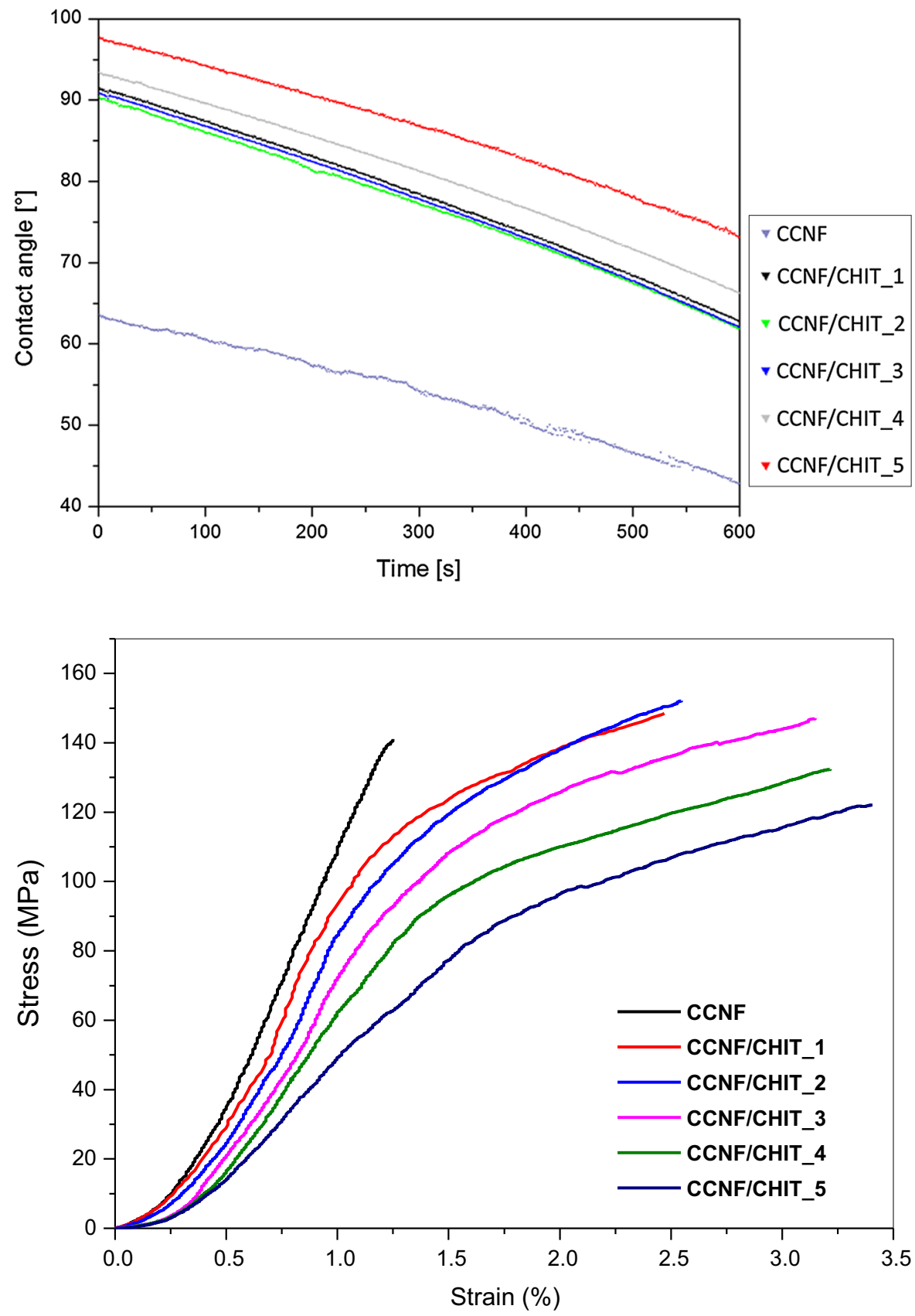

range of $16.02-38.63 \%$ for $E$. coli and $2.70-11.63 \%$ for $S$. epidermidis was observed. An inhibition effect for Micrococcus luteus was obtained only for CCNF/ CHIT_3 with approximately $11 \%$ of the inhibition zone. By contrast, only a weak inhibition effect was observed on the fungi. Because previous reports showed the inhibition effect of chitosan alone on bacteria, chitosan was tested at a higher concentration (at $0.25 \%$ and $1 \%$ ). The obtained results indicated that Petriella setifera, Botrytis cinerea, and Neosartorya fischeri degraded the chitosan added to the medium. Particularly, B. cinerea and $N$. fischeri degraded the chitosan at both concentrations $(0.25 \%$ and $1 \%)$, whereas $P$. setifera degraded chitosan only at $0.25 \%$ (Supplementary Materials Fig. 4). During the incubation period, a different behaviour of these 
Table 3 Mechanical properties of CCNF/CHIT composites

\begin{tabular}{lcllll}
\hline Samples & $\begin{array}{l}\text { Young's modulus } \\
(\mathrm{GPa})\end{array}$ & $\begin{array}{l}\text { Yield strength } \\
(\mathrm{MPa})\end{array}$ & Yield strain $(\%)$ & $\begin{array}{l}\text { Tensile strength } \\
(\mathrm{MPa})\end{array}$ & Maximum strain (\%) \\
\hline CCNFs & $14.71 \pm 1.27$ & $92.90 \pm 15.05$ & $0.83 \pm 0.09$ & $145.84 \pm 37.66$ & $1.35 \pm 0.35$ \\
CCNF/CHIT_1 & $10.82 \pm 2.00$ & $78.65 \pm 12.18$ & $0.96 \pm 0.12$ & $141.37 \pm 22.80$ & $2.68 \pm 0.80$ \\
CCNF/CHIT_2 & $10.89 \pm 1.02$ & $78.58 \pm 10.23$ & $0.95 \pm 0.12$ & $139.53 \pm 20.27$ & $2.08 \pm 0.55$ \\
CCNF/CHIT_3 & $9.68 \pm 0.88$ & $79.65 \pm 11.60$ & $0.98 \pm 0.17$ & $138.50 \pm 19.01$ & $2.35 \pm 0.58$ \\
CCNF/CHIT_4 & $8.82 \pm 0.79$ & $70.54 \pm 6.36$ & $0.97 \pm 0.10$ & $129.28 \pm 15.75$ & $2.76 \pm 0.79$ \\
CCNF/CHIT_5 & $8.76 \pm 1.10$ & $76.37 \pm 6.04$ & $1.09 \pm 0.09$ & $129.43 \pm 15.06$ & $2.68 \pm 0.68$ \\
\hline
\end{tabular}

Table 4 Percent of the inhibition zone with the standard deviation for each tested microorganism $(\mathrm{n}=3)$

\begin{tabular}{lcccccc}
\hline & CCNFs & CCNF/ & CCNF/ & CCNF/ & CCNF/ & \multicolumn{1}{l}{ CCNF/ } \\
CHIT_1 & CHIT_2 & CHIT_3 & CHIT_4 & CHIT_5 \\
\hline Bacillus cereus & $1.95 \pm 0.00$ & $1.46 \pm 0.72$ & $1.95 \pm 0.00$ & $1.95 \pm 0.00$ & $1.95 \pm 0.00$ & $1.95 \pm 0.00$ \\
Botrytis cinerea & $1.75 \pm 2.49$ & $0.87 \pm 1.40$ & $1.26 \pm 1.63$ & $1.80 \pm 2.60$ & $1.65 \pm 2.95$ & $4.03 \pm 5.64$ \\
Candida krissii & $0.00 \pm 0.00$ & $0.00 \pm 0.00$ & $0.00 \pm 0.00$ & $0.00 \pm 0.00$ & $0.00 \pm 0.00$ & $0.61 \pm 1.17$ \\
Escherichia coli & $\mathbf{3 5 . 5 2} \pm \mathbf{2 2 . 5 3}$ & $\mathbf{3 7 . 8 9} \pm \mathbf{3 5 . 6 3}$ & $\mathbf{3 8 . 6 3} \pm \mathbf{2 9 . 9 4}$ & $\mathbf{1 6 . 0 2} \pm \mathbf{8 . 2 7}$ & $\mathbf{2 9 . 6 4} \pm \mathbf{3 0 . 2 9}$ & $\mathbf{3 4 . 0 1} \pm \mathbf{3 2 . 1 0}$ \\
Fusarium oxysporum & $1.26 \pm 1.63$ & $0.97 \pm 1.02$ & $1.13 \pm 1.58$ & $1.60 \pm 1.23$ & $2.48 \pm 1.60$ & $3.16 \pm 2.13$ \\
Micrococcus luteus & $1.30 \pm 1.54$ & $1.83 \pm 3.40$ & $1.46 \pm 0.88$ & $\mathbf{1 0 . 6 8} \pm \mathbf{1 5 . 7 1}$ & $3.18 \pm 3.73$ & $\mathbf{8 . 3 4} \pm \mathbf{1 4 . 1 9}$ \\
Neosartorya fischeri & $0.81 \pm 1.00$ & $1.14 \pm 1.00$ & $1.30 \pm 0.96$ & $0.49 \pm 0.88$ & $0.49 \pm 0.88$ & $0.49 \pm 0.88$ \\
Petriella setifera & $0.81 \pm 1.00$ & $1.14 \pm 1.00$ & $1.14 \pm 1.00$ & $2.68 \pm 2.68$ & $0.49 \pm 0.88$ & $1.36 \pm 1.90$ \\
Staphylococcus & $\mathbf{1 1 . 6 3} \pm \mathbf{1 8 . 4 5}$ & $\mathbf{7 . 1 1} \pm \mathbf{6 . 7 9}$ & $2.70 \pm 1.32$ & $\mathbf{7 . 1 0} \pm \mathbf{6 . 8 1}$ & $\mathbf{1 0 . 4 8} \pm \mathbf{1 4 . 7 4}$ & $3.59 \pm 2.50$ \\
epidermidis & & & & & & \\
\hline
\end{tabular}

For each microorganisms are seen the average percentage of the inhibition zone of three replication plus the deviation standard. The highest inhibition zones were bolded for each composite

microorganisms from the optimal growing condition was observed. In the case of Bacillus cereus, we saw that the bacterial growth on chitosan was different than that of the control. B. cereus growing on chitosan created more colonies and did not cover all the plates as that in the control (Supplementary Materials Fig. 5a). The same effect was observed for E. coli (Supplementary Materials Fig. 5b). By contrast, for the fungi, in general, the inhibition effect of cellulose nanofibrils and chitosan was not observed. A short inhibition zone of these two compounds after 1 day of incubation of Fusarium oxysporum and Botrytis cinerea was noted (Supplementary Materials Fig. 6).
Antimicrobial assay through the optical density (OD) measurement

Considering the type of composite (Fig. 5a), it was observed that the control (microorganisms that grew in normal and optimal conditions) presented the highest OD value, whereas the lowest was recorded for the CCNF/CHIT_4 and CCNF/CHIT_5 films. By contrast, the pure $\mathrm{CCNF}$ and $\mathrm{CHIT}$ caused average microorganism growth but a visible decrease in the $\mathrm{OD}$ value. The trend of OD versus incubation time (Fig. 5b) showed that the decrease in the OD value was obtained for all tested composites after the first $24 \mathrm{~h}$. Moreover, for CHIT alone, OD was stable over time. However, the CCNF and CCNF/CHIT composites decreased and reached stable values after $72 \mathrm{~h}$. 
(a)

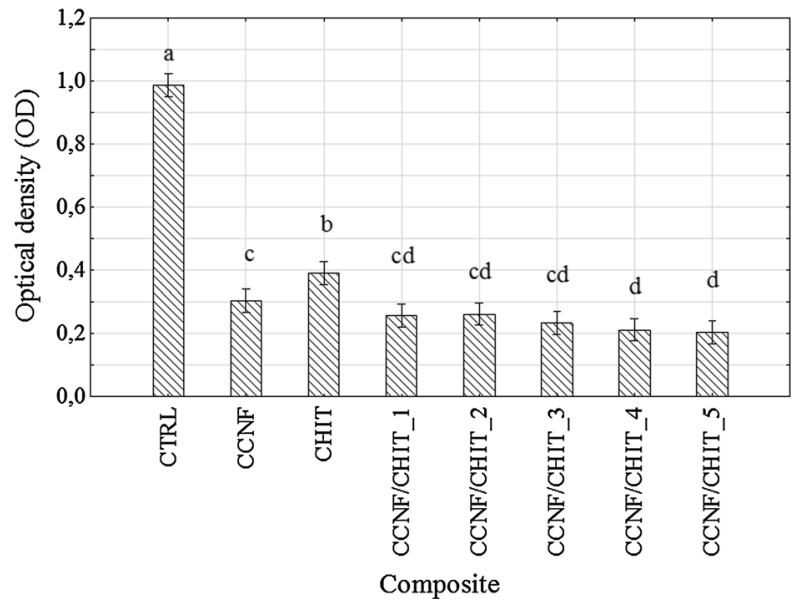

Fig. 5 Average optical density (OD) for all tested bacteria and fungi measured until 7 days of incubation at $37{ }^{\circ} \mathrm{C}$ or $28^{\circ} \mathrm{C}$ as a function of each treatment (a) and sampling time (b). The

Table 5 Significant differences between the average optical density (OD) of all microorganisms for all tested composites (including nanocellulose and chitosan alone)

\begin{tabular}{ll}
\hline Microorganisms & Average OD \\
\hline Bacillus cereus & $0.313^{\mathrm{c}}$ \\
Botrytis cinerea & $0.196^{\mathrm{d}}$ \\
Candida krissii & $0.232^{\mathrm{c}, \mathrm{d}}$ \\
Escherichia coli & $0.483^{\mathrm{b}}$ \\
Fusarium oxysporum & $0.164^{\mathrm{d}}$ \\
Micrococcus luteus & $0.329^{\mathrm{c}}$ \\
Neosartorya fischeri & $0.337^{\mathrm{c}}$ \\
Petriella setifera & $0.635^{\mathrm{a}}$ \\
Staphylococcus epidermidis & $0.538^{\mathrm{a}, \mathrm{b}}$ \\
\hline
\end{tabular}

Superscript letters denote the significant differences (Post-hoc Tukey's test $p$ value $<0.05$ )

Analysing the optical density (Table 5), a different behaviour of each microorganism was noted. The high value of OD indicates that bacteria or fungi grow normally under the analysed conditions (Fig. 5). It was observed that only Botrytis cinerea, Fusarium oxysporum and Candida krissii were inhibited by the tested composites, findings that were confirmed statistically.

The $0.25 \%$ CHIT used in the incubation medium caused a decrease in OD at the beginning of the incubation time. However, after $72 \mathrm{~h}$, the OD values increased and obtained a value higher than that (b)

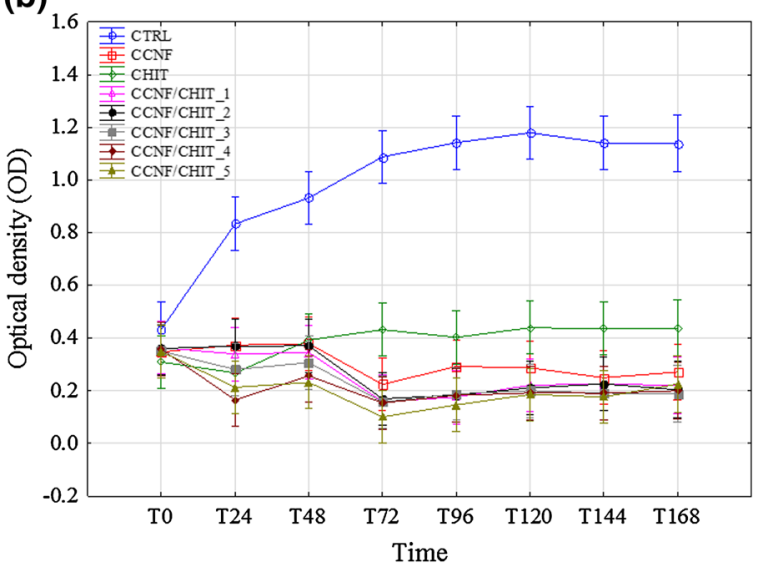

vertical bars indicate the confidence intervals at 0.95 , and the lowercase letters indicate the significant differences $(p<0.05)$ calculated using post hoc Tukey's test

obtained for the composites under study. Analysing the OD of $1 \%$ chitosan, it was found that the OD value for all microorganisms increased until the 4th day of incubation and then reached a stable value at approximately 0.9 until the end of the experiment (Fig. 6a). Figure $6 \mathrm{~b}$ shows that, for the duration of the experiment, the OD of the control was higher (c.a. twotimes more) than that observed in the chitosan treatment.

\section{Discussion}

The composites based on nanocellulose prepared from carrot cellulose with different concentrations of chitosan from shrimp shells were prepared. The optical transparency of films was very good only for the film with the highest content of chitosan (CCNF/CHIT_5) starts to become opaque. The deterioration of the transparency, in this case, could be the result of the aggregation of CCNFs (Toivonen et al. 2015). Additionally, SEM micrographs showed no difference between composites and CCNF film surfaces, indicating the proof of obtaining a homogenous mixture of chitosan and nanocellulose. Toivonen et al. (2015) showed that nanocellulose cross-linked with chitosan formed tough films with good optical properties. The interaction between CCNFs and CHIT was evaluated in terms of FTIR spectra. Even though the mechanism of interaction between nanocellulose and chitosan is 


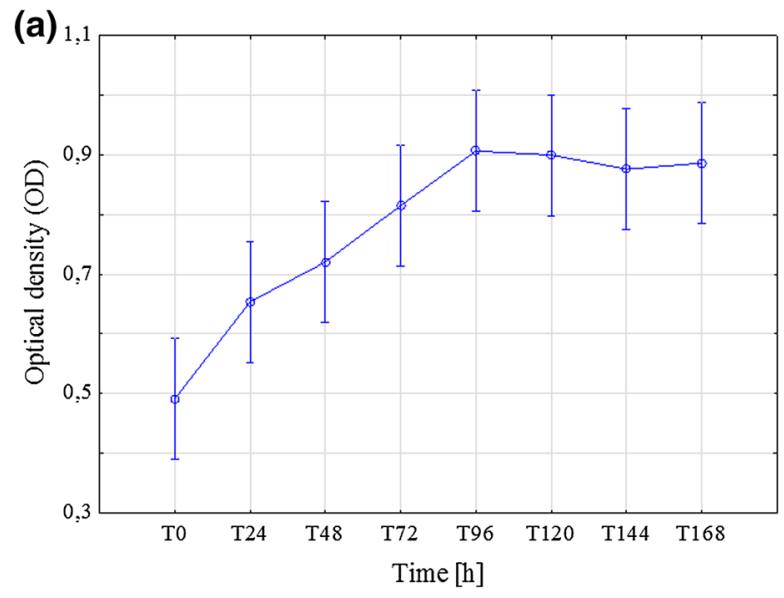

Fig. 6 Optical density (OD) for all tested fungi measured until 7th day of incubation at $28{ }^{\circ} \mathrm{C}$ as a function of sampling time: average OD for all samples (a) and OD for control and fungi

still not fully understood the most probable are hydrogen or ionic interactions, covalent imine linkage, and also hydrophobic interactions (Toivonen et al. 2015). The most pronounced changes in the FTIR spectra should be related to hydrogen bonding represented by bonds at approximately $3600-3000 \mathrm{~cm}^{-1}$ (Khan et al. 2012). However, no differences in the spectra region mentioned above were observed for the CCNF/CHIT composites and compared with the pure CCNFs and CHIT spectra. This lack of differences allows the assumption that there was likely no hydrogen bonding between nanocellulose and chitosan. On the other hand, the FTIR spectra of the CCNF/CHIT composites showed that only bands related to amide II and amide I were slightly changed. Previously, the redshift of amide bands i.e., towards lower wavenumbers was related to the interaction between nanocellulose and chitosan ( $\mathrm{Hu}$ et al. 2015). Moreover, the disappearance of the ester band at $1740 \mathrm{~cm}^{-1}$, characteristic for oxidized CCNFs, could suggest the interaction between the oxidized surface of cellulose and amide groups of chitosan. Toivonen et al. (2015) suggested that crosslinking between cellulose nanofibres and chitosan is based on a physical interaction between the reducing ends of cellulose and primary amines of chitosan, promoting the dehydration of chitosan. This finding has been supported by the water wettability experiment. Nanocellulose is known to have poor water resistance and low water contact angle. Tangpasuthadol et al. (2003) showed that water contact angle of chitosan

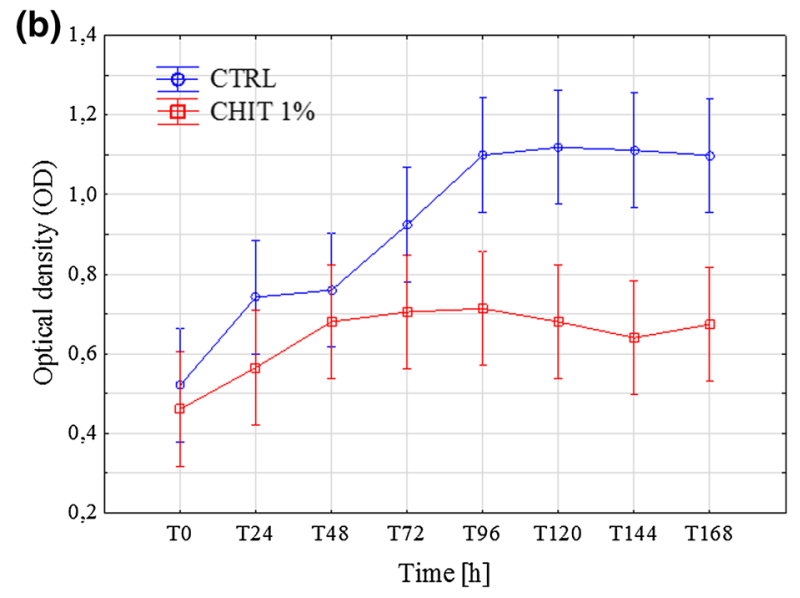

incubated in presence of $1 \%$ chitosan inside inoculum (b). The vertical bars indicate the confidence intervals at 0.95

films is around $89 \pm 6^{\circ}$ which is much higher than value obtained in this study for CCNF films. The addition of chitosan to the nanocellulose matrix causes an increase in the water contact angle i.e., the surface of the composites become more hydrophobic. This increase could be connected to the interaction between nanocellulose and chitosan resulting in a denser structure. The degradation temperatures ca. $300{ }^{\circ} \mathrm{C}$ are similar to those obtained by Jia et al. (2017) using the TGA method. High degradation temperatures assure that the composites are not affected by the temperature (Ostadhossein et al. 2015). Additionally, none of the peak temperatures matches the temperatures of decomposition of pure CCNFs or CHIT, implying interactions between these polymers in composites (Jia et al. 2017). Moreover, the addition of chitosan to the nanocellulose matrix caused an increase in the degradation process enthalpy.

The addition of chitosan to CCNF matrices caused a decrease in Young's moduli the higher concentration of chitosan in the composite leads to a lower Young's modulus. The yield strength describes the maximum force that can be applied for reversible deformation. The addition of chitosan caused a decrease in the yield strength of the composites. Additionally, the tensile strength of composites i.e., the maximum force that causes a fracture was decreased after the addition of chitosan. Only the strain had the tendency to increase after chitosan addition. These results slightly differed from those that could be found in the literature. Toivonen et al. (2015) analysed the cellulose 
nanofibres-chitosan composites, and they found that chitosan improved the mechanical properties of the composites compared with the pure nanocellulose film. On the other hand, most of the papers reported changes in the mechanical properties of composites where the nanocellulose is additive to the chitosan matrix (Wu et al. 2014; Fernandes et al. 2010). Generally, nanocellulose was an additive of the chitosan matrix improved the mechanical properties of composites. Wu et al. (2014) found that cellulose nanofibrils incorporated in chitosan films produced by the solution casting method caused a significant increase in the tensile strength and Young's modulus. However, Li et al. (2009) reported that, by increasing the cellulose nanowhisker concentration from 0 to $20 \mathrm{wt} \%$, the tensile strength of the films raised from 85 to $120 \mathrm{MPa}$ for dry composites and from 9.9 to 17.3 $\mathrm{MPa}$ for wet chitosan composites. It was also observed that the incorporation of cellulose nanowhiskers enhanced water resistance and the thermal stability of chitosan films.

The main purpose of using chitosan as an additive to films was to exploit its antimicrobial properties. The microorganisms chosen for antimicrobial testing were either human pathogens (Staphylococcus epidermidis, Escherichia coli, Bacillus cereus, Micrococcus luteus, Candida krissii) or plant pathogens (Botrytis cinerea, Neosartorya fischeri, Petriella setifera and Fusarium oxysporum). The greatest inhibition was observed for Escherichia coli and Staphylococcus epidermidis. Micrococcus luteus was inhibited at the highest chitosan concentration in film. Moreover, for Bacillus cereus and $E$. coli, modification of their growth mode was observed. Benhabiles et al. (2012) discovered that chitosan can lead to a change in the gene expression in Staphylococcus aureus SG511. Consequently, the modification in the growing modes seen in Supplementary materials Fig. 5 could be the effect of chitosan exposure. The disk diffusion analysis revealed that the combination of cellulose nanofibrils and chitosan has a strong effect on Escherichia coli and Staphylococcus epidermidis, confirming the effect of chitosan on both Gram-positive and Gram-negative bacteria (Benhabiles et al. 2012; Dutta et al. 2009; Hosseinnejad and Jafari 2016; Leceta et al. 2013; Poonguzhali et al. 2017). The fungal cultures were resistant to the chitosan presence in films. However, disk diffusion analysis showed that, after 1 day of incubation, an inhibition zone around the film was observed (Supplementary materials Fig. 4 and 6). Subsequently, this inhibition zone decreased with the experimental duration, and finally, the mycelium completely covered the film. This behaviour of the fungal culture can be connected to the fluidity of the cell membrane because the increase in the antimicrobial activity of chitosan is connected to the increase in the amount of unsaturated fatty acids in the cell membrane (Verlee et al. 2017). This outcome could be the reason for the fungal resistance to the presence of chitosan.

Additionally, chitosan dispersed in incubation medium was used as the control experiment for the antimicrobial properties of chitosan alone. The degradation of the chitosan inside the Petri dish was observed only for Botrytis cinerea, Neosartorya fischeri, and Petriella setifera (Supplementary materials Fig. 4). Chitosan degradation can be obtained through chitinases (E.C. 3.2.1.14), chitosanases (E.C. 3.2.1.132) (Zhang and Neau 2001), and exo-glucosaminidase (E.C. 3.2.1.165) (Nidheesh et al. 2015). Chitonase enzyme is produced from bacteria (e.g., Myxobacter, Sporocytophaga, Arthrobacter, Bacillus, and Streptomyces) and fungi (Rhizopus, Aspergillus, Penicillium, Chaetomium, and Basidiomycetes that are very rich in glucanase) (Gooday 1990). Thus, it could be deduced that $B$. cinerea, $N$. fischeri, and P. setifera likely produced some of these enzymes and chitosan is simply a source of carbon used by fungi, explaining their resistance to the CCNF/CHIT composites.

The optical density (OD) was used to evaluate the antimicrobial activity of the CCNF/CHIT composites. The OD value obtained for all tested microorganisms in the presence of CCNF/CHIT composites was lower than that of the control samples (Figs. 5, 6). Additionally, both concentrations of chitosan (1\% and $0.25 \%$ ) had OD values lower than that of the control sample (Figs. 5, 6), indicating that the composites had bacteriostatic and fungistatic activities on the analysed microorganisms; however, they did not kill analysed microorganisms completely. Particularly, during the incubation time, we saw a significant decrease in the OD value of the analysed film after $72 \mathrm{~h}$. Chitosan, in contact with water, most likely exerted antimicrobial activity against all the analysed microorganisms, reducing the growth density (measured as optical density). Furthermore, only $P$. setifera presented a higher OD; this result could be connected to the presence of $\beta$-glucosidase. Zhang and Neau (2001) 
studied the $\beta$-glucosidase activity on chitosan and found that the higher the degree of deacetylation (DD) of chitosan is, the slower is its degradation. In that study, the preparation of $\beta$-glucosidase also contained chitinase. P. setifera studied here could degrade chitosan through the utilization of both enzymes. Moreover, the analysis of OD values indicates that chitosan alone has less efficiency than that of the CCNF/CHIT composite; the combination of chitosan with cellulose nanofibrils could improve the antimicrobial activity of chitosan on bacteria and fungi. Additionally, studies on the bacterio- or fungistatic properties of chitosan, which is soluble in an acidic environment, are usually conducted at low $\mathrm{pH}$ (Badawy and Rabea 2009). In the present study, although chitosan was dissolved in acidic solution, the CCNF/CHIT composites were neutralized (Schillinger and Lücke 1989). In this way, we analysed the real effect of chitosan-cellulose nanofibril composites on bacteria and fungi without the influence of low $\mathrm{pH}$.

\section{Conclusions}

Films of carrot cellulose nanofibrils (CCNFs) with the addition of low-viscosity chitosan (CHIT) were prepared by the vacuum filtration method. The chitosan content in the films was varied from 9 (CCNF/ CHIT_1) to $33 \%$ (CCNF/CHIT_5) of dry wt. The optical transparency of films was very good except for those with the highest content of chitosan. The deterioration of the transparency, in this case, could be the result of the aggregation of CCNFs. SEM showed that chitosan was dispersed homogeneously in the CCNF matrix. FTIR spectroscopy suggested that there was interaction between the oxidized surface of the CCNFs and the amide groups of CHIT. This finding is also supported in the water wettability experiment. The addition of chitosan to the nanocellulose matrix causes an increase in composite hydrophobicity. This increase is probably connected with the interaction between nanocellulose and chitosan to form a denser structure. Analyses of the thermal properties showed that composites are stable at high temperature, and the degradation occurred above $300{ }^{\circ} \mathrm{C}$. It was found that the addition of chitosan to CCNF matrices caused a decrease in Young's moduli, indicating that chitosan acts as a plasticizer and enhances the ductility of nanocellulose itself. Additionally, the tensile strength of composites decreased after the addition of chitosan. The analysed antimicrobial properties indicated the highest inhibitory effect of cellulose nanofibrils-chitosan composite films against $E$. coli and S. epidermidis. It should also be highlighted that this is the first work analysing the antimicrobial properties of nanocellulose-chitosan composites without the influence of acetic acid present in composites to dissolve chitosan. Therefore, we can confirm that the composites of carrot nanocellulose with the addition of chitosan has antimicrobial activity, especially fungistatic and bacteriostatic activity.

Acknowledgments The work was partially funded by The National Centre for Research and Development-Poland (Grant No. LIDER/300/L-6/14/NCBR/2015).

Author's contribution MSC conceived and designed the experiment, wrote the manuscript, and obtained and analysed the FTIR and Raman spectra. MSC and MC designed and performed the experiments involving cellulose isolation and nanocellulose preparation. MC performed and analysed the DSC curves and contact angle and participated in the results and discussions. GP and MF designed, performed and discussed the results of microbiological tests. PMP performed the mechanical tests and was involved in the analysis of their results. KJC obtained SEM micrographs. AZ helped in manuscript preparation.

\section{Compliance with ethical standards}

Conflict of interest The authors declared that they have no conflict of interest.

Open Access This article is distributed under the terms of the Creative Commons Attribution 4.0 International License (http:// creativecommons.org/licenses/by/4.0/), which permits unrestricted use, distribution, and reproduction in any medium, provided you give appropriate credit to the original author(s) and the source, provide a link to the Creative Commons license, and indicate if changes were made.

\section{References}

Abdul Khalil HPS, Saurabh CK, Adnan AS, Nurul Fazita MR, Syakir MI, Davoudpour Y, Rafatullah M, Abdullah CK, Haafiz MKM, Dungani R (2016) A review on chitosancellulose blends and nanocellulose reinforced chitosan biocomposites: properties and their applications. Carbohydr Polym 150:216-226

Abo-Elseoud WS, Hassan ML, Sabaa MW, Basha M, Hassan EA, Fadel SM (2018) Chitosan nanoparticles/cellulose nanocrystals nanocomposites as a carrier system for the 
controlled release of repaglinide. Int $\mathrm{J}$ Biol Macromol 111:604-613

Alemdar A, Sain M (2008) Isolation and characterization of nanofibers from agricultural residues-wheat straw and soy hulls. Bioresour Technol 99:1664-1671

Ämmälä A, Liimatainen H, Burmeister C, Niinimäki J (2013) Effect of TEMPO and periodate-chlorite oxidized nanofibrils on ground calcium carbonate flocculation and retention in sheet forming and on the physical properties of sheets. Cellulose 20:2451-2460

Azeredoa HMC, Rosaa MF, Mattoso LHC (2017) Nanocellulose in bio-based food packaging applications. Ind Crops Prod 97:664-671

Badawy MEI, Rabea EI (2009) Potential of the biopolymer chitosan with different molecular weights to control postharvest gray mold of tomato fruit. Postharvest Biol Technol 51:110-117

Benhabiles MS, Salah R, Lounici H, Drouiche N, Goosen MFA, Mameri N (2012) Antibacterial activity of chitin, chitosan and its oligomers prepared from shrimp shell waste. Food Hydrocoll 29:48-56

Brinchia L, Cotana F, Fortunati E, Kenny JM (2013) Production of nanocrystalline cellulose from lignocellulosic biomass: technology and applications. Carbohydr Polym 94:154-169

Chen Y (2017) Preparation and application of the composite of chitosan. In: Thakur VK, Thakur MK, Kessler MR (eds) Handbook of composites from renewable materials, structure and chemistry, vol 1. Wiley, New York, pp 371-433

Chen W, Yu H, Liu Y, Chen P, Zhang M, Hai Y (2011) Individualization of cellulose nanofibers from wood using high-intensity ultrasonication combined with chemical pretreatments. Carbohydr Polym 83:1804-1811

de Alvarenga ES (2011) Characterization and properties of chitosan. In: Elnashar M (ed) Biotechnology of biopolymers. InTech, Rijeka, pp 91-108

de Mesquita JP, Donnici CL, Pereira FV (2010) Biobased nanocomposites from layer- by-layer assembly of cellulose nanowhiskers with chitosan. Biomacromolecules 11(2):473-480

de Souza Costa Júnior E, Pereira MM, Mansur HS (2009) Properties and biocompatibility of chitosan films modified by blending with PVA and chemically crosslinked. J Mater Sci Mater Med 20:553-561

Dehnad D, Mirzaei H, Emam-Djomeh Z, Jafari S-M, Dadash S (2014) Thermal and antimicrobial properties of chitosannanocellulose films for extending shelf life of ground meat. Carbohydr Polym 109:148-154

Deng Z, Jung J, Zhao Y (2017) Development, characterization, and validation of chitosan adsorbed cellulose nanofiber (CNF) films as water resistant and antibacterial food contact packaging. LWT Food Sci Technol 83:132-140

Dimzon IKD, Knepper TP (2015) Degree of deacetylation of chitosan by infrared spectroscopy and partial least squares. Int J Biol Macromol 72:939-945

Dufresne A (2012) Cellulose and potential reinforcement. In: Dufresne A (ed) Nanocellulose: from nature to high performance tailored materials. De Gruyter, Berlin, pp 1-37
Dutta PK, Tripathi S, Mehrotra GK, Dutta J (2009) Perspectives for chitosan based antimicrobial films in food applications. Food Chem 114:1173-1182

Elsabee MZ, Abdou ES (2013) Chitosan based edible films and coatings: a review. Mater Sci Eng C 33:1819-1841

Falamarzpour P, Behzad T, Zamani A (2017) Preparation of nanocellulose reinforced chitosan films, cross-linked by adipic acid. Int J Mol Sci 18:396

Fernandes SCM, Freire CSR, Silvestre AJD, Pascoal Neto C, Gandini A, Berglund L, Salmén L (2010) Transparent chitosan films reinforced with a high content of nanofibrillated cellulose. Carbohydr Polym 81:394-401

Gooday GW (1990) Physiology of microbial degradation of chitin and chitosan. Biodegradation 1:177-190

Hänninen A, Sarlin E, Lyyra I, Salpavaara T, Kellomäki M, Tuukkanen S (2018) Nanocellulose and chitosan based films as low cost, green piezoelectric materials. Carbohydr Polym 202:418-424

Hassan ML, Hassan EA, Oksman KN (2011) Effect of pretreatment of bagasse fibers on the properties of chitosan/ microfibrillated cellulose nanocomposites. J Mater Sci 46:1732-1740

Hassan EA, Hassan ML, Abou-zeid RE, El-Wakil NA (2016) Novel nanofibrillated cellulose/chitosan nanoparticles nanocomposites films and their use for paper coating. Ind Crops Prod 93:219-226

Hosseinnejad M, Jafari SM (2016) Evaluation of different factors affecting antimicrobial properties of chitosan. Int $\mathrm{J}$ Biol Macromol 85:467-475

Hu CX, Yang G, Zhang Q, Chen F, Fu Q (2015) Mechanically reinforced chitosan/cellulose nanocrystals composites with good transparency and biocompatibility. Chin J Polym Sci 33(1):61-69

Hudzicki J (2009) Kirby-Bauer disk diffusion susceptibility test protocol. American Society for Microbiology, ASM Microbe Library. http://www.asmscience.org/content/ education/protocol/protocol.3189

Jia Y, Wang X, Huo M, Zhai X, Li F, Zhong C (2017) Preparation and characterization of a novel bacterial cellulose/chitosan bio-hydrogel. Nanomater Nanotechnol 7:1-8

Khan RA, Salmieri S, Dussault D, Calderon JU, Kamal MR, Safrany A, Lacroix M (2010) Production and properties of nanocellulose reinforced methylcellulose based biodegradable films. J Agric Food Chem 58(13):7878-7885

Khan A, Khan RA, Salmieri S, Le TC, Riedl B, Bouchard J, Chauve G, Tan V, Kamal MR, Lacroix M (2012) Mechanical and barrier properties of nanocrystalline cellulose reinforced chitosan based nanocomposite films. Carbohydr Polym 90(4):1601-1608

Kim KW, Min BJ, Kim Y-T, Kimmel RM, Cooksey K, Park SI (2011) Antimicrobial activity against foodborne pathogens of chitosan biopolymer films of different molecular weights. LWT Food Sci Technol 44(2):565-569

Klemm D, Kramer F, Moritz S, Lindström T, Ankerfors M, Gray D, Dorris A (2011) Nanocelluloses: a new family of naturebased materials. Angew Chem Int Ed 50(24):5438-5466

Kong M, Chen XG, Xing K, Park HJ (2010) Antimicrobial properties of chitosan and mode of action: a state of the art review. Int J Food Microbiol 144:51-63 
Krishna Rao KSV, Vijaya Kumar Naidu B, Subha MCS, Sairam M, Aminabhavi TM (2006) Novel chitosan based pH sensitive interpenetrating network microgels for the controlled release of cefadroxil. Carbohydr Polym 66:333-344

Lavoine N, Desloges I, Dufresne A (2012) Microfibrillated cellulose-its barrier properties and applications in cellulosic materials: a review. Carbohydr Polym 90:735-764

Leceta I, Guerrero P, Ibarburu I, Dueñas MT, De la Caba K (2013) Characterization and antimicrobial analysis of chitosan-based films. J Food Eng 116:889-899

Li Q, Renneckar S (2011) Supramolecular structure characterization of molecularly thin cellulose I nanoparticles. Biomacromolecules 12:650-659

Li Q, Zhou JP, Zhang LN (2009) Structure and properties of the nanocomposite films of chitosan reinforced with cellulose whiskers. J Polym Sci Polym Phys 47:1069-1077

Li X, Shi X, Wang M, Du Y (2011) Xylan chitosan conjugate-a potential food preservative. Food Chem 126(2):520-525

Liu B, Zhang J, Liu L, Hotchkiss AT (2012) Utilization of pectin extracted sugar beet pulp for composite application. J Biobased Mater Bioenergy 6:185-192

Moon RJ, Martini A, Nairn J, Simonsen J, Youngblood J (2011) Cellulose nanomaterials review: structure, properties and nanocomposites. Chem Soc Rev 40:3941-3994

Nada AMA, Hassan ML (2000) Thermal behaviour of cellulose and some cellulose derivatives. Polym Degrad Stab 67:111-115

Nidheesh T, Kumar PG, Suresh PV (2015) Enzymatic degradation of chitosan and production of D-glucosamine by solid substrate fermentation of exo- $\beta$-D-glucosaminidase (exochitosanase) by Penicillium decumbens CFRNT15. Int Biodeterior Biodegrad 97:97-106

Niu X, Liu Y, Song Y, Han J, Pan H (2018) Rosin modified cellulose nanofiber as a reinforcing and co-antimicrobial agents in polylactic acid/chitosan composite film for food packaging. Carbohydr Polym 183:102-109

Ostadhossein F, Mahmoudi N, Morales-Cid G, Tamjid E, Navas-Martos FJ, Soriano-Cuadrado B, Paniza JML, Simchi A (2015) Development of chitosan/bacterial cellulose composite films containing nanodiamonds as a potential flexible platform for wound dressing. Materials 8:6401-6418

Poonguzhali R, Basha SK, Kumari VS (2017) Synthesis and characterization of chitosan-PVP-nanocellulose composites for in vitro wound dressing application. Int J Biol Macromol 105:111-120

Rahmi L, Julinawati S (2017) Preparation of chitosan composite film reinforced with cellulose isolated from oil palm empty fruit bunch and application in cadmium ions removal from aqueous solutions. Carbohydr Polym 170:226-233

Rambabu N, Panthapulakkal S, Sain M, Dalai AK (2016) Production of nanocellulose fibers from pinecone biomass: evaluation and optimization of chemical and mechanical treatment conditions on mechanical properties of nanocellulose films. Ind Crops Prod 83:746-754

Schillinger U, Lücke F-K (1989) Antibacterial activity of Lactobacillus sake isolated from meat. Appl Environ Microb 55:1901-1906

Stark NM (2016) Opportunities for cellulose nanomaterials in packaging films: a review and future trends. J Renew Mater 4(5):313-326
Szymańska-Chargot M, Zdunek A (2013) Use of FT-IR Spectra and PCA to the bulk characterization of cell wall residues of fruits and vegetables along a fraction process. Food Biophys 8(1):29-42

Szymańska-Chargot M, Cybulska J, Zdunek A (2011) Sensing the structural differences in cellulose from apple and bacterial cell wall materials by Raman and FT-IR spectroscopy. Sensors 11:5543-5560

Szymańska-Chargot M, Chylińska M, Gdula K, Kozioł A, Zdunek A (2017) Isolation and characterization of cellulose from different fruit and vegetable pomaces. Polymers 9:495

Szymańska-Chargot M, Cieśla J, Chylińska M, Gdula K, Pieczywek PM, Kozioł A, Cieślak KJ, Zdunek A (2018) Effect of ultrasonication on physicochemical properties of apple based nanocellulose-calcium carbonate composites. Cellulose 25:4603-4621

Szymańska-Chargot M, Chylińska M, Pieczywek PM, Zdunek A (2019) Tailored nanocellulose structure depending on the origin. Example of apple parenchyma and carrot root celluloses. Carbohydr Polym 210:186-195

Tangpasuthadol V, Pongchaisirikul N, Hoven VP (2003) Surface modification of chitosan films: effects of hydrophobicity on protein adsorption. Carbohydr Res 338(9):937-942

Toivonen MS, Kurki-Suonio S, Schacher FH, Hietala S, Rojas OJ, Ikkala O (2015) Water-resistant, transparent hybrid nanopaper by physical cross-linking with chitosan. Biomacromolecules 16:1062-1071

Trana CD, Duria S, Delnerib A, Franko M (2013) Chitosancellulose composite materials: preparation, characterization and application for removal of microcystin. J Hazard Mater 252-253:355-366

Verlee A, Mincke S, Stevens CV (2017) Recent developments in antibacterial and antifungal chitosan and its derivatives. Carbohydr Polym 164:268-283

Wang W, Sabo RC, Mozuch MD, Kersten P, Zhu J, Jin Y (2015) Physical and mechanical properties of cellulose nanofibril films from bleached eucalyptus pulp by endoglucanase treatment and microfluidization. J Polym Environ 23:551-558

Wu T, Farnood R, O’Kelly K, Chen B (2014) Mechanical behaviour of transparent nanofibrillar cellulose-chitosan nanocomposite films in dry and wet conditions. J Mech Behav Biomed 32:279-286

Zhang H, Neau SH (2001) In vitro degradation of chitosan by a commercial enzyme preparation: effect of molecular weight and degree of deacetylation. Biomaterials 22:1653-1658

Zhbankov RG, Firsov SP, Buslov DK, Nikonenko NA, Marchewka MK, Ratajczak H (2002) Structural physicochemistry of cellulose macromolecules. Vibrational spectra and structure of cellulose. J Mol Struct 614:117-125

Publisher's Note Springer Nature remains neutral with regard to jurisdictional claims in published maps and institutional affiliations. 